Ann. Biol. anim. Bioch. Biophys., I96I, 1 (3) 305-310

\title{
LES VARIATIONS DE LA COMPOSITION MINERALE DU LAIT DE VACHE
}

\author{
L. GUEGUEN et M. JOURNET \\ Avec la collaboration technique de Michelle LaNGLOIs. \\ Service de Biochimie et de Nutrition et Station de Recherches sur l'Elevage, \\ Centre national de Recherches zootechniques, Jouy-en-Josas (S-et-O.).
}

\section{SOMMAIRE}

L'étude des variations des teneurs en $\mathrm{P}, \mathrm{Ca}, \mathrm{K}, \mathrm{Na}, \mathrm{Mg}$ du lait au cours de la lactation a été effectuée sur dix vaches de trois races différentes, ayant toutes vêlé au début de l'hiver et ayant reçu le même régime alimentaire.

Les dosages ont porté sur un échantillon moyen hebdomadaire provenant de toutes les traites de la semaine.

$\mathrm{Au}$ cours de la lactation les teneurs en $\mathrm{K}$ diminuent régulièrement tandis que les teneurs en $\mathrm{Na}$ augmentent, surtout quelques semaines avant le tarissement. Les teneurs en $\mathrm{Ca}$ et $\mathrm{P}$ sont maxima au début et en fin de lactation.

Le lait des vaches Normandes semble plus riche en $\mathrm{Ca}$ et plus pauvre en $\mathrm{K}$ que le lait des vaches de races Frisonne et Pie rouge de l'Est.

La mise au pâturage n'influe pas sur la composition minérale du lait.

La composition minérale du lait de vache a déjà fait l'objet de multiples travaux et ses variations sont assez bien connues. Toutefois, plusieurs facteurs de variation pouvant interférer, il a souvent été difficile de réunir des conditions expérimentales permettant de faire agir un seul facteur.

Nous avons étudié l'influence du stade de lactation sur les teneurs en $\mathrm{K}, \mathrm{Na}, \mathrm{P}$, $\mathrm{Ca}$ et $\mathrm{Mg}$ du lait de dix vaches soumises au même régime alimentaire et ayant vêlé à la même époque. Ceci a donc permis d'éliminer les différences entre animaux dues à l'alimentation et à la saison.

Les animaux ayant reçı une nourriture constamment adaptée à leurs besoins, nous pensons avoir réduit au minimum l'influence des facteurs alimentaires et enregistré au mieux l'évolution physiologique de la composition minérale du lait de vaches ayant vêlé en hiver. D'autre part, l'utilisation d'un échantillon moyen pondéré de toutes les traites de chaque semaine atténue l'influence des variations journalières.

\section{MATÉRIEL E'T MÉTHODES}

\section{Animaux}

Les dix vaches utilisées (5 Frisonnes Pie noires, 3 Normandes et 2 Pie rouges de l'Esst) ont vêlé de la mi-Novembre à la fin de décembre r958. Le tableau I en donne les caractéristiques essentielles. 
TABI,EAU I

Liste et caractéristiques des vaches utilisées

\begin{tabular}{|c|c|c|c|c|}
\hline Vache No & Lactation $\mathrm{N}^{\circ}$ & Date de Vêlage & $\begin{array}{l}\text { Durée de la lac- } \\
\text { tation (jours) }\end{array}$ & $\begin{array}{l}\text { Production de } \\
\quad \text { lait }(\mathrm{kg})\end{array}$ \\
\hline Normandes & & & & \\
\hline 24 & 3 & $15-11-58$ & 329 & 3819 \\
\hline 267 & 3 & $27-11-58$ & 275 & 3413 \\
\hline 268 & 4 & $29-11-58$ & 238 & $299^{\prime}$ \\
\hline \multicolumn{5}{|l|}{ Frisonnes } \\
\hline 30 & 5 & $11-12-58$ & 277 & 3301 \\
\hline 37 & 4 & $26-12-58$ & 365 & 4863 \\
\hline 197 & 3 & $31-12-58$ & 262 & 3500 \\
\hline 207 & 3 & $31-12-58$ & 381 & 5069 \\
\hline 211 & 3 & $18-12-58$ & 208 & 2980 \\
\hline \multicolumn{5}{|l|}{ Pie rouges } \\
\hline 246 & 2 & $14-12-58$ & 315 & 3717 \\
\hline 247 & 3 & $7-12-58$ & $244^{\prime}$ & 2965 \\
\hline
\end{tabular}

Par lactation, la production moyenne de lait a été de $3662 \mathrm{~kg}$ avec des extrêmes de 2465 à $5069 \mathrm{~kg}$; la teneur moyenne en matiéres grasses a été de $37,95 \mathrm{~g}$ par $\mathrm{kg}$ et la teneur en matières azotées de $34, \mathrm{I} 7 \mathrm{~g}$ par $\mathrm{kg}$. Le coefficient de persistance de la production laitière entre la $3^{\mathrm{e}}$ et la $3^{\mathrm{o}^{\mathbf{e}} \mathrm{se}-}$ maine, a été de 89,6 .

L'état sanitaire des animaux a été satisfaisant du vêlage au tarissement. Toutefois, deux cas de mammite ont été enregistrés, avec infection des 4 quartiers durant toute la lactation pour la vache $\mathrm{n}^{0} 30$ (deux épisodes aigus suivis de traitement au cours du $3^{\mathrm{e}}$ et $\mathrm{du} 4^{\mathrm{e}}$ mois de lactation) et infection d'un seul quartier pour la vache $\mathrm{n}^{0} 207$.

\section{Régime alimentaire}

En hiver, les animaux ont reçu $20 \mathrm{~kg}$ de betteraves à forte teneur en matière sèche, de l'ensilage d'herbe et du foin (graminées + légumineuses) à volonté. La mise à l'herbe (pâturage rationné sur mélange de graminées et légumineuses) a eu lieu le I4 avril I959. Les besoins des animaux, selon leur format et leur production laitière, ont été couverts par l'apport d'un concentré constitué de céréales, de tourteaux et d'un composé minéral $(65 \mathrm{p}$. roo de phosphate bicalcique et $35 \mathrm{p}$. roo de sel). Au cours des six semaines précédant le vêlage, chaque animal a reçu en moyenne $2 \mathrm{~kg}$ de concentré par jour.

\section{Prélèvement et conservation des échantillons}

Les vaches ont été traites deux fois par jour et le lait pesé individuellement à chaque traite. A partir des prélèvements effectués à chacune de ces traites nous avons constitué des échantillons pondérés sur une semaine. Après addition de I $\mathrm{ml}$ d'une solution $\left.{ }^{1}\right)$ de sublimé pour $\mathrm{r} 60 \mathrm{ml}$ de lait, les échantillons ont été placés en chambre froide à o- $2^{\circ} \mathrm{C}$. Cette teneur de $0,4 \mathrm{~g}$ de $\mathrm{HgCl}_{2}$ par litre de lait ne perturbe pas les dosages.

\section{Méthodes d'analyse}

Les dosages ont été effectués sur le résidu de minéralisation nitro-perchlorique d'une prise d'essai de $5 \mathrm{ml}$ de lait, traitée par $10 \mathrm{ml}$ de mélange nitro-perchlorique (2 parties de $\mathrm{HClO}_{4}+\mathrm{I}$ partie de $\mathrm{HNO}_{3}$ ) dans un matras d'attaque chauffé sur un bain de sable à $220-250^{\circ} \mathrm{C}$.

Après décoloration du mélange (au bout de 4 à 5 heures) on a repris par environ $20 \mathrm{ml}$ d'eau bi-distillée et on a laissé bouillir $3^{\circ}$ minutes environ. Puis, après refroidissement, on a transvasé dans

(1) La solution de sublimé est ainsi constituée : $64 \mathrm{~g}$ de $\mathrm{HgCl}_{2}, 500 \mathrm{ml}$ d'alcool et $500 \mathrm{ml}$ d'eau distillée. 
des fioles jaugées de $5 \circ \mathrm{ml}$. Le dosage de $\mathrm{Mg}$ a été effectué directement sur cette solution (lait $\mathrm{I} / \mathrm{IO}$ ). et les dosages de $\mathrm{P}, \mathrm{K}, \mathrm{Na}$ et $\mathrm{Ca}$ après dilution de $\mathrm{I} / \mathrm{I}$ o (lait $\mathrm{I} / 5^{\circ}$ ).

Le phosphore a été dosé par la méthode colorimétrique au jaune de phosphovanadomolybdate d'ammonium et les métaux alcalins et alcalino-terreux par spectrophotométrie de flamme (GUEGUEN et Rombauts 196r).

\section{RÉSULTATS}

\section{Io Teneurs moyennes pour des lactations complètes}

Le tableau 2 indique les teneurs moyennes individuelles en éléments minéraux, calculées sur toute la durée de la lactation, et les valeurs moyennes obtenues pour l'ensemble des Io vaches avec les écarts-types correspondants. Les teneurs individuelles ne sont pas simplement les moyennes arithmétiques de tous les résultats hebdomadaires, mais sont des moyennes pondérées calculées en tenant compte des variations de la production laitière. Ainsi, les teneurs indiquées pour l'ensemble des ro vaches peuvent être considérées comme représentatives d'un " lait de mélange ".

TABLEAU 2

Teneurs moyennes pondérées en éléments minéraux du lait (pour des lactations complètes) en $\mathrm{g}$ par $\mathrm{kg}$.

\begin{tabular}{|c|c|c|c|c|c|}
\hline Vache No & $\mathrm{P}$ & $\mathrm{Ca}$ & $\mathrm{K}$ & $\mathrm{Na}$ & $\mathrm{Mg}$ \\
\hline Normandes & & & & & \\
\hline 24 & 0,92 & 1,38 & 1,55 & 0,39 & 0,14 \\
\hline 267 & 0,96 & 1,45 & 1,45 & $0,4.3$ & 0,13 \\
\hline 268 & 0,91 & 1,37 & 1,32 & 0,40 & 0,13 \\
\hline Frisonnes & & & & & \\
\hline 30 & 1,02 & 1,35 & 1,62 & 0,54 & 0,15 \\
\hline 37 & 0,89 & 1,16 & 1,49 & 0,61 & $0,1^{\prime}$ \\
\hline 197 & 0,92 & 1,31 & 1,70 & 0,49 & 0,1 \\
\hline 207 & 0,97 & 1,13 & 1,80 & 0,45 & 0,14 \\
\hline 211 & 0,87 & 1,11 & 1,77 & 0,48 & 0,14 \\
\hline Pie rouges & & & & & \\
\hline 246 & 0,90 & 1,21 & 1,73 & 0,38 & $0,1^{\prime} t$ \\
\hline 247 & 0,90 & 1,22 & 1,76 & 0,35 & 0,13 \\
\hline Moyennes $\left( \pm \sigma_{m}\right)$ & $0,93 \pm 0,01$ & $1,27 \pm 0,04$ & $1,6: \pm 0,05$ & $0,47 \pm 0,02$ & 0,14 \\
\hline
\end{tabular}

Les différences individuelles sont très accentuées pour $\mathrm{K}, \mathrm{Ca}$ et $\mathrm{Na}$, et beaucoup moins pour $\mathrm{P}$ et $\mathrm{Mg}$.

Il n'est malheureusement pas possible, sur un nombre aussi limité d'individus, d'insister sur les différences observées entre races. Toutefois, nous pouvons signaler que le lait de la race Normande semble plus riche en Ca que le lait des deux autres races ( $\mathrm{I}, 40 \mathrm{~g}$ par $\mathrm{kg}$ contre $\mathrm{I}, 22 \mathrm{~g}$ par $\mathrm{kg}$ en moyenne); ceci peut être lié à la teneur plus élevée en caséine du lait de la race Normande. Les teneurs élevées en Ca semblent être généralement associées à des teneurs faibles en $\mathrm{K}$ (le lait des vaches Normandes est relativement pauvre en $\mathrm{K}$ ). D'autre part, il ne semble pas y avoir de relation entre le taux butyreux et les teneurs en éléments minéraux du lait. 
Enfin, nous nous sommes demandés si les fortes productions laitières ne provoquaient pas, comme pour d'autres constituants, une « dilution 》 des éléments minéraux dans le lait. En fait, il n'en est rien et les exportations sont sensiblement proportionnelles aux productions laitières. Les exportations totales d'éléments minéraux durant toute la lactation sont très variables d'un individu à l'autre : de $2.503 \mathrm{~g}$ à $4.663 \mathrm{~g}$ pour $\mathrm{P}$, de $3.248 \mathrm{~g}$ à $5.525 \mathrm{~g}$ pour $\mathrm{Ca}$, de $3.862 \mathrm{~g}$ à $9 . \mathrm{I} 24 \mathrm{~g} \mathrm{~K}$ et de I. I27g à 3 .o $5 \mathrm{~g}$ pour Na.

\section{0) Influence du stade de lactation}

La figure suivante rassemble les teneurs moyennes hebdomadaires en $\mathrm{P}, \mathrm{Ca}$, $\mathrm{K}, \mathrm{Na}$ et $\mathrm{Mg}$ du lait des ro vaches de $1 \mathrm{a} 2^{\mathrm{e}}$ à $\mathrm{la} 3^{\mathrm{I}}$ semaine de lactation, puis du lait de 7 et enfin de 4 vaches de la $33^{\mathrm{e}}$ à la $45^{\mathrm{e}}$ semaine (3 des vaches utilisées étaient taries at1 bout de 7 mois).

Fait bien connu, les teneurs en $\mathrm{K}$ et Na évoluent en sens inverse ; 1'augmentation de la teneur en $\mathrm{Na}$ n'est toutefois sensible qu'à partir de la $30^{\mathrm{e}}$ semaine environ, tandis que les teneurs en $\mathrm{K}$ diminuent régulièrement au cours de la lactation de $\mathrm{I}, 8$ à $\mathrm{I}, 3 \mathrm{~g}$ par $\mathrm{kg}$. Seuls les laits de fin de lactation ont des teneurs élevées en $\mathrm{Na}(0,6$ à $0,7 \mathrm{~g}$ par kg).

Le colostrum n'a pas été étudié, mais il est bien connu que chez la vache il est beaucoup plus riche que le lait normal en $\mathrm{P}$ et $\mathrm{Ca}$, dont les teneurs diminuent ensuite rapidement au cours des premières semaines de lactation. A partir du $2^{\mathrm{e}}$ mois de lactation la teneur en $\mathrm{Ca}$ varie peu, puis elle semble augmenter de nouveau en fin de lactation (ce qui est sans doute lié à l'accroissement de la teneur en caséine) ; les teneurs en $\mathrm{K}$ et $\mathrm{Ca}$ sont alors voisines.

La teneur en $\mathrm{P}$ diminue sensiblement au cours de la lactation et semble passer par un minimum vers le $6^{\mathrm{e}}$ mois.

Enfin, les teneurs en Mg, très faibles, varient peu avec le stade de lactation.

\section{$3^{\circ}$ Influence de la mise à l'herbe}

Nous avons rassemblé dans le tableau 3 les teneurs moyennes hebdomadaires en éléments minéraux du lait des dix vaches étudiées pour les six semaines entourant la mise à l'herbe. Compte tenu de l'importance des variations physiologiques nor-

TABLEAU 3

Influence de la mise à l'herbe sur la composition minérale du lait (en g par $\mathrm{kg}$ ).

\begin{tabular}{|c|c|c|c|c|c|c|}
\hline & \multicolumn{3}{|c|}{ Avant la mise à l'herbe } & \multicolumn{3}{|c|}{ Après la mise à l'herbe } \\
\hline & $\begin{array}{c}3 \text { semaines } \\
\text { avant }\end{array}$ & $\begin{array}{c}2 \text { semaines } \\
\text { avant }\end{array}$ & $\begin{array}{l}1 \text { semaine } \\
\text { avant }\end{array}$ & $\begin{array}{l}1 \text { semaine } \\
\text { après }\end{array}$ & $\begin{array}{c}2 \text { semaines } \\
\text { après }\end{array}$ & $\begin{array}{l}3 \text { semaines } \\
\text { après }\end{array}$ \\
\hline $\mathrm{P} \ldots \ldots$ & 0,93 & 0,92 & 0,93 & 0,95 & 0,86 & 0,92 \\
\hline $\mathrm{Ca} \ldots . . \mathrm{C}$ & 1,23 & 1,16 & 1,22 & 1,29 & 1,27 & 1,24 \\
\hline $\mathrm{K} \ldots$ & 1,66 & 1,70 & 1,63 & 1,70 & 1,68 & 1,63 \\
\hline $\mathrm{Na} . . .$. & 0,45 & $0,{ }_{4} 6$ & 0,44 & 0,43 & 0,45 & 0,44 \\
\hline $\operatorname{Mg} \ldots$ & 0,13 & 0,14 & 0,13 & 0,14 & 0,15 & 0,14 \\
\hline
\end{tabular}




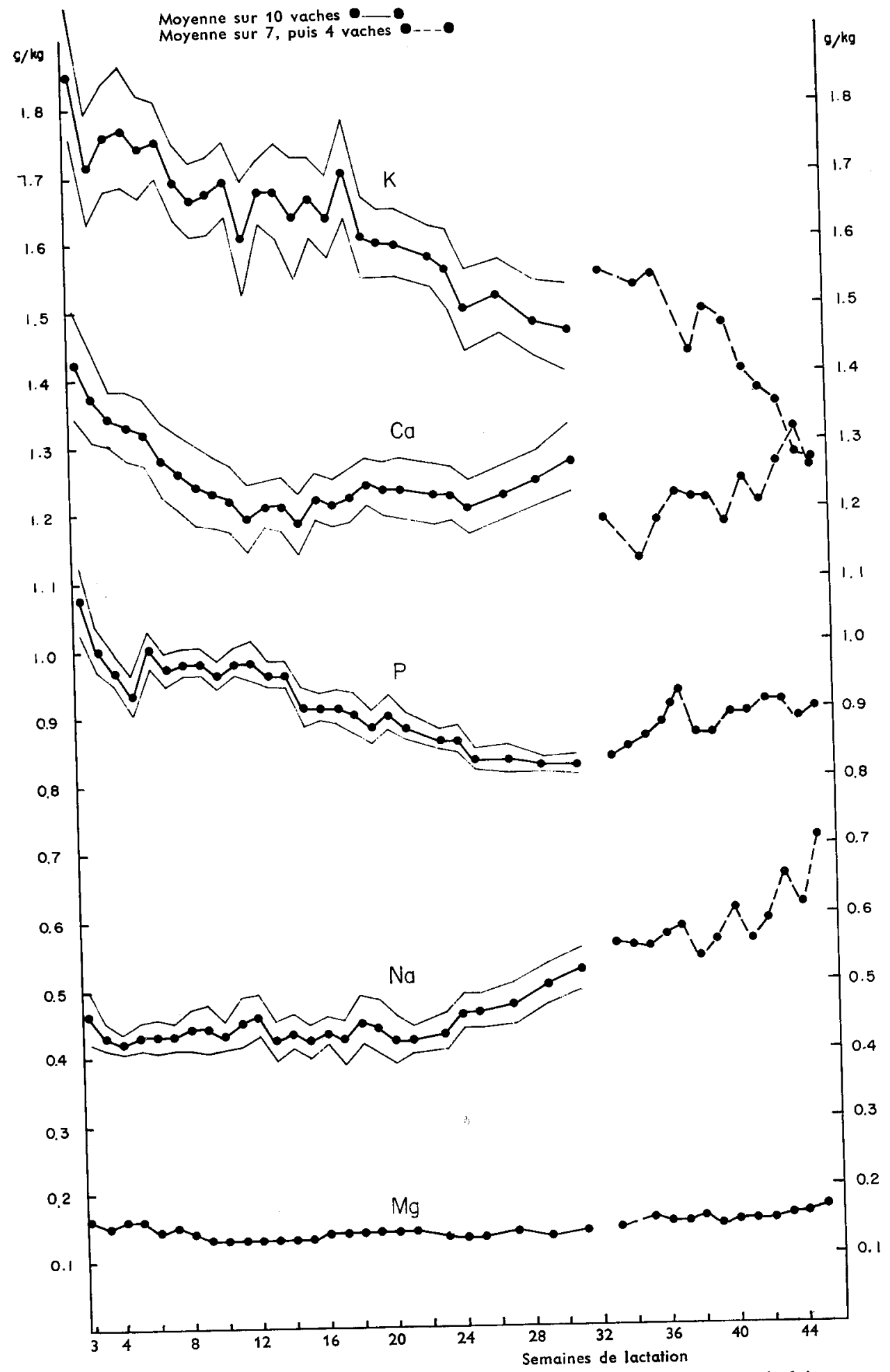

FIG. I. - Influence du stade de lactation sur les teneurs en éléments minéraux du lait.

(L'écart type affectant chaque moyenne est représenté sur le graphique de part et d'autre de la courbe de variation).

Annales de Biologie Animale. - I96r. 
males des teneurs en $\mathrm{P}$ et $\mathrm{Ca}$ du lait à cette époque (au moment de la mise à l'herbe les animaux se trouvaient entre $1 \mathrm{a} I 5^{\mathrm{e}}$ et $1 \mathrm{a} 20^{\mathrm{e}}$ semaine de lactation) il est difficile d'attribuer à l'alimentation les légères variations observées pour ces deux éléments. Dans l'ensemble, cette variation brutale du régime alimentaire n'a pas d'influence sur la composition minérale du lait.

\section{$4^{\circ}$ Influence de l'infection microbienne de la mamelle}

Le lait moyen de la vache $n^{\circ} 30$ présentant une infection généralisée aux 4 quar_ tiers, a une teneur en $\mathrm{Na}$ supérieure de $20 \mathrm{p}$. Ioo à la teneur moyenne. Les épisodes aigus ont été suivis d'un accroissement très net de la teneur en $\mathrm{Na}$ du lait, qui revient à son niveau normal après le traitement. En revanche, les épisodes aigus ont provoqué une diminution importante et passagère de la teneur en $\mathrm{K}$ du lait. Ces teneurs anormales pourraient donc être dues à une dilution du lait par transsudation de plasma sanguin.

Il convient de signaler que les teneurs observées et leurs variations ne sont sans doute valables que pour des vaches ayant vêlé au début de la période de stabulation. En effet, VANSCHOUBROECK (I958) a montré que la saison pouvait avoir une influence sur la composition du lait de vache et surtout sur les teneurs en $\mathrm{P}$ et $\mathrm{Ca}$, qui semblent en général plus faibles en été, à la fin de la période de pâturage. En revanche les teneurs en $\mathrm{Mg}$ sont les plus faibles au début de l'hiver. Toutefois, malgré l'existence de cette action saisonnière, les principaux facteurs de variation de la composition minérale du lait de vache sont manifestement l'individu et le stade de lactation.

Reçu en décembre 1960.

\section{SUMMARY}

\section{VARIATIONS IN MINERAL COMPOSITION OF COW'S MILK}

The study of variations in the $\mathrm{P}, \mathrm{Ca}, \mathrm{K}, \mathrm{Na}$ and $\mathrm{Mg}$ contents of milk in the course of lactation has been carried out with Io cows of 3 different breeds which had all calved at the beginning of the winter and which had all received the same diet. The determinations were carried out on a weekly sample obtained from all the milkings of the week using the vanadate colorimetric method for $\mathrm{P}$, and flame spectrophotometry for $\mathrm{Ca}, \mathrm{K}, \mathrm{Na}$ and $\mathrm{Mg}$ after nitro-perchloric digestion.

In the course of lactation, the $\mathrm{K}$ content diminishes regularly whereas the Na content increases, especially at the end of lactation. The $\mathrm{Ca}$ and $\mathrm{P}$ contents are highest at the beginning and end of lactation.

The milk from Normandy cows appears to be richer in $\mathrm{Ca}$ and poorer in $\mathrm{K}$ than the milk from Fresian and Eastern Red Pied cows.

The mineral composition of the milk is not affected if the animals are put out to pasture.

\section{RÉFÉRENCES BIBLIOGRAPHIQUES}

Gueguen L., Rovbauts P., igor. Dosage du sodium, du potassium, du calcium et du magnésium par spectrophotométrie de flamme dans les aliments, le lait et les excreta. Ann. Biol. anim. Bioch. Biophys., 1, 80-97.

VANschoubroeck F. X., 1958. Seasonal influences on the yield and composition of cow's milt. Nederl. Melk Zuivell. 12, 1 2-37. 